Check for updates

Cite this: RSC Adv., 2018, 8, 8800

Received 25th January 2018

Accepted 16th February 2018

DOI: 10.1039/c8ra00770e

rsc.li/rsc-advances

\title{
Oxide-assisted growth of scalable single-crystalline graphene with seamlessly stitched millimeter-sized domains on commercial copper foils $\uparrow$
}

\begin{abstract}
Yang Wang, ${ }^{a}$ Yu Cheng, ${ }^{a}$ Yunlu Wang, ${ }^{a}$ Shuai Zhang, ${ }^{b}$ Xuewei Zhang, ${ }^{a}$ Shaoqian Yin, ${ }^{a}$ Miao Wang, ${ }^{c}$ Yang Xia, ${ }^{d}$ Qunyang Li, ${ }^{b}$ Pei Zhao (D) *a and Hongtao Wang*a

Chemical vapor deposition (CVD) is considered as an effective route to obtain large-area and high-quality polycrystalline graphene; however, there are still technological challenges associated with its application to achieve single crystals of graphene. Herein, we present the CVD growth of scalable single-crystalline graphene by seamless stitching millimeter-sized unidirectional aligned hexagonal domains using different types of commercial $\mathrm{Cu}$ foils without repeated substrate polishing and $\mathrm{H}_{2}$-annealing processes. Compared with that reported in previous studies, herein, the average size for the hexagonal graphene domains is enlarged by 1-2 orders of magnitude (from tens of micrometers to millimeter). The key factor for growth is the $\mathrm{Cu}$ surface monocrystallization achieved by a pre-introduced oxide layer and the sequential $\mathrm{Ar}$ annealing. The graphene domains exhibit an average growth rate of $>20 \mu \mathrm{m} \mathrm{min}{ }^{-1}$ and a misorientation possibility of $<2 \%$, and seamless stitching at the domain coalescence interfaces is confirmed by atomic force microscopy measurements.
\end{abstract}

\section{Introduction}

Copper (Cu)-based chemical vapor deposition (CVD) has been standing out as the-state-of-the-art technique in the scalable production of graphene due to the easy access to the monolayer and its excellent quality. ${ }^{1-3}$ However, graphene grown by this technique tends to have polycrystalline structures because of the nucleation and growth of domains with different in-plane orientations, and the resultant defective domain boundaries probably degrade the electronic quality, mechanical strength, and thermal conductivity of graphene. ${ }^{4-7}$ Therefore, the growth of scalable single-crystalline graphene without domain boundaries should be a key step for the advancement of the CVD technique. Current efforts to achieve single-crystalline graphene growth focus on two possible strategies. The first strategy involves the suppression of the nucleation density of graphene to avoid the possible formation of domain boundaries and obtain the graphene domains as large as possible. However, in this strategy, the growth normally requires a long period of up

${ }^{a}$ Institute of Applied Mechanics, Key Laboratory of Soft Machines and Smart Devices of Zhejiang Province, Zhejiang University, Hangzhou 310012, P. R. China. E-mail: peizhao@zju.edu.cn; htw@zju.edu.cn

${ }^{b}$ Center for Nano and Micro Mechanics, Applied Mechanics Laboratory, School of Aerospace Engineering, Tsinghua University, Beijing 100084, P. R. China

'Department of Physics, Zhejiang University, Hangzhou 310012, P. R. China

${ }^{d}$ Institute of Microelectronics, Chinese Academy of Science, Beijing 100029, P. R. China

$\dagger$ Electronic supplementary information (ESI) available: Supplementary Figures. See DOI: $10.1039 / \mathrm{c} 8 \mathrm{ra} 00770 \mathrm{e}$ to tens of hours. ${ }^{8-12}$ The other strategy involves a precise control of the orientations of the graphene domain and a seamless stitching of the adjacent domains using a single-crystalline metal substrate such as $\mathrm{Cu}(111)$ and $\mathrm{Cu}(100)$. However, the attempts based on this approach are often frustrated by the complication and cost of the $\mathrm{Cu}$ surface treatments such as tenuous polishing and/or elongated annealing, tightly rolled $\mathrm{Cu}$ foils into a cylinder, etc. ${ }^{13-18}$ In a recent study reported by Xu et al., the epitaxial growth of meter-sized single-crystal graphene was realized, but a relatively complex experimental setup with small constituent domain sizes $(<100 \mu \mathrm{m})$ was used, making the probability of the coalesced domain boundaries not negligible even with a $99 \%$ unidirectional orientation. ${ }^{19}$

Herein, we demonstrated a new pathway for the growth of scalable monolayer single-crystalline graphene by seamlessly stitching the unidirectionally aligned arrays of millimeter-sized hexagonal domains using different types of commercially available $\mathrm{Cu}$ foils and industrially safe atmospheric pressure conditions. Compared with that reported in similar previous studies, ${ }^{13-19}$ the average size for the hexagonal graphene domains is enlarged by $1-2$ orders of magnitude (from tens of micrometers to millimeter); this further eliminates the formation of domain boundaries. The key factor that leads to the success of this growth is the monocrystallization of the catalytic $\mathrm{Cu}$ surface to centimeter-sized (111) textures by simply preintroducing a $\mathrm{Cu}$ oxide layer for high-temperature surface reconstruction. We believe that this study will bring an important step forward towards the industrial production of highquality graphene for devices and other applications. 


\section{Results and discussion}

The growth procedure of the aHGDs is illustrated in Fig. 1a. A commercially available $\mathrm{Cu}$ foil (\#46365 from Alfa Aesar China Chemical Co., Ltd.) is simply heated at $200-350{ }^{\circ} \mathrm{C}$ for $10-$ $30 \mathrm{~min}$ in the air to form an oxide layer before it is placed into a CVD chamber. The sequent short-time hydrogen $\left(\mathrm{H}_{2}\right)$-free annealing process reconstructs the $\mathrm{Cu}$ foil surface for a centimeter scale monocrystallization to the (111) texture. Although the X-ray diffraction (XRD, Fig. 1b) spectra show that both the pristine and annealed $\mathrm{Cu}$ foils are dominated by the (100) texture, further investigation on the annealed $\mathrm{Cu}$ foil by electron backscatter diffraction (EBSD, Fig. 1c) clearly reveals the presence of a homogenous surface with the (111) texture over a large area. Considering that EBSD reflects more structural information within the several-nanometer depth near the crystal surface, it is clear that a uniform thin $\mathrm{Cu}$ (111) layer is formed on top of the pristine polycrystalline $\mathrm{Cu}$ foil. Fig. 1d displays an image of the as-grown graphene domains on the reconstructed $\mathrm{Cu}$ foil with the control parameter (pre-oxidation at $300{ }^{\circ} \mathrm{C}$ for $30 \mathrm{~min}$ and $60 \mathrm{~min}$ growth). The color contrast of graphene is realized by slightly oxidizing the as-grown $\mathrm{Cu}$ foil in the air. ${ }^{20}$ The sizes of these graphene domains are approximately $0.5-2.5$ millimeters, indicating a maximum growth rate of higher than $40 \mu \mathrm{m} \mathrm{min}{ }^{-1}$. The scanning electron microscopy (SEM) image of the graphene domains with a shorter growth period of 0.5 hour is shown in Fig. 1e. All the graphene domains observed in the image are unidirectionally aligned on the $\mathrm{Cu}$ surface with a misorientation angle of less than $1^{\circ}$ as well as a hexagonal shape with straight edges. Similar results of these aligned hexagonal graphene domains (aHGDs) were reproduced on other types of commercial $\mathrm{Cu}$ foils (Fig. S1, ESI $\dagger$ ), indicating that the oxide-assisted surface monocrystallization was irrelevant to the $\mathrm{Cu}$ production process. It also needs to be noted that the growth results of these aHGDs are not influenced by a prolonged annealing process with $\mathrm{H}_{2}$; this indicates that a changed reduction gas atmosphere does not affect the established surface monocrystallinity of $\mathrm{Cu}$ (Fig. S2, ESI $\dagger$ ). Moreover, the growth rate of a graphene film formed by aHGDs can be tuned by modifying the $\mathrm{Cu}$ pre-oxidation parameters (i.e., temperature and time) for different nucleation densities of the domain, as shown in Fig. S3 in ESI. $\dagger$ Fig. 1f shows an optical microscopy (OM) image of a graphene sample, whose domains are on the verge of totally coalescing together into a global continuous film, on $\mathrm{Cu}$ with the maximum film growth rate (pre-oxidation at $205{ }^{\circ} \mathrm{C}$ for $10 \mathrm{~min}$ and $40 \mathrm{~min}$ growth). The uncovered $\mathrm{Cu}$ regions (i.e., dark orange areas) are surrounded by the straight graphene domain edges that are parallel to each other or with an included angle of $60^{\circ}$ or $120^{\circ}$; this confirms the unidirectional alignment of the coalesced graphene domains to which these edges belong. The formed continuous graphene film exhibits a sheet resistance of $\sim 270 \pm 30 \Omega \square^{-1}$, close to the value reported by Xu et al. for their meter-scale graphene single crystals. ${ }^{19}$ We have performed Raman spectroscopy with a $532 \mathrm{~nm}$ excitation laser on the aHGDs transferred onto the $\mathrm{SiO}_{2} / \mathrm{Si}$ wafers. All of their typical Raman spectra exhibit the fingerprint of monolayer graphene with a high intensity ratio of the $2 \mathrm{D}\left(\sim 2690 \mathrm{~cm}^{-1}\right)$ to $\mathrm{G}\left(\sim 1580 \mathrm{~cm}^{-1}\right)$ bands, a narrow and symmetric $2 \mathrm{D}$ band, and a negligible D-band $\left(\sim 1340 \mathrm{~cm}^{-1}\right.$, originated from the structural defects of graphene), ${ }^{21}$ as shown in Fig. 1g, indicating the very high quality of these aHGDs.

The uniformity and quality of the aHGDs were further determined by the scanning Raman maps of the intensities for the G, 2D, and D bands (Fig. 2). The graphene domain appears in a uniform contrast when inspected by OM except for the small sites due to the polymer residues from the transfer process; this indicates that the layer number is uniform across the sample. Correspondingly, in the Raman maps, most

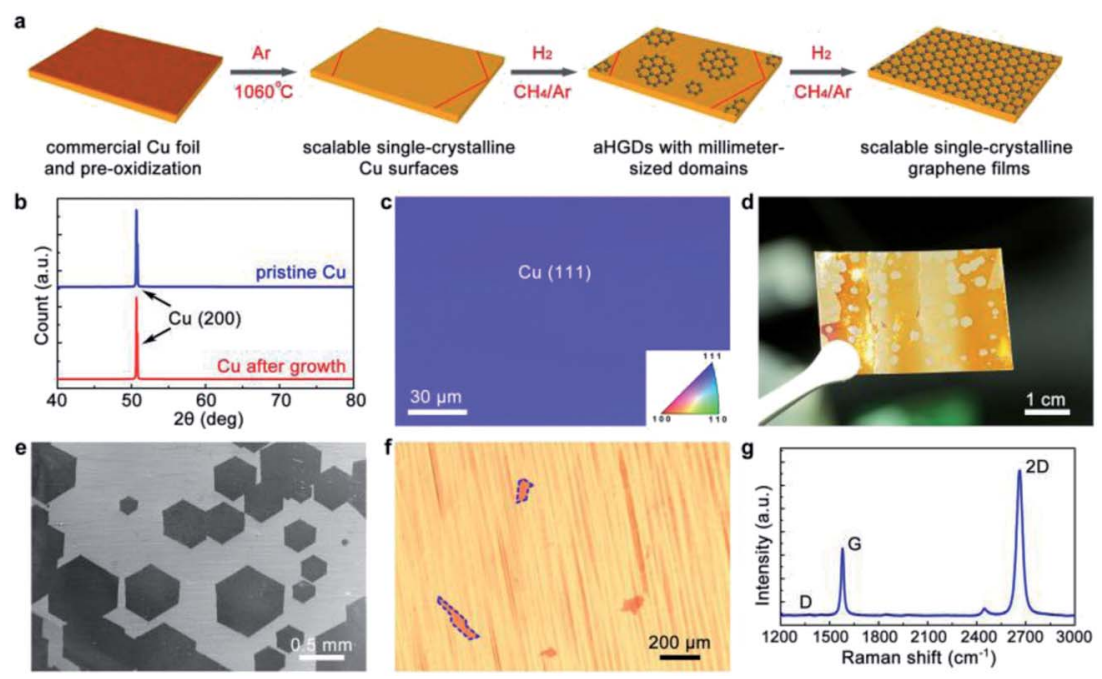

Fig. 1 (a) Schematic of the CVD growth procedure. (b) The XRD measurements show that the Cu substrates before and after graphene growth are dominated by the (100) texture. (c) The EBSD results demonstrate an actual surface monocrystallization to the (111) texture for the Cu substrates. (d) An optical image for individual aHGDs grown for 60 min. (e) An SEM image for individual aHGDs grown for 30 min. (f) An OM image of a nearly continuous graphene film coalesced by aHGDs. The exposed Cu surfaces (dark orange areas) confirm the alignment of coalesced aHGDs. (g) Typical Raman spectrum of aHGDs indicates the monolayer nature and high quality of the sample. 

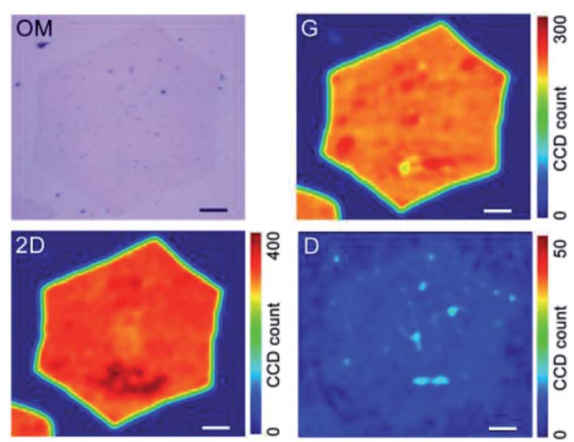

Fig. 2 The OM image and scanning Raman spectroscopy results for the $G, 2 D$, and $D$ bands for an aHGD transferred onto the $\mathrm{SiO}_{2} / \mathrm{Si}$ substrate. Scale bars: $100 \mu \mathrm{m}$.

scanned areas of the graphene domain exhibit uniform $\mathrm{G}$ and 2D band intensity distributions except for the domain edges, and the average $2 \mathrm{D}$-to-G intensity ratio is $\sim 1.8 \pm 0.3$, indicative of the monolayer nature for the whole domain. Moreover, the Raman spectra in the whole domain present relatively low D band intensities including the domain edges, which not only demonstrate the overall high quality of the graphene but also, to some extent, help confirm the zigzag edge structure for these domains; this has been further discussed hereinafter.

Fig. 3 further presents the alignment of these graphene domains on the $\mathrm{Cu}$ foil in more details by scanning the domains within a large view field $\left(10.5 \times 7.5 \mathrm{~mm}^{2}\right)$ with a $5 \times$ microscope objective and organizing the $16 \mathrm{OM}$ images together. A different growth condition with a shorter pre-oxidation and growth time is adopted in the sample to increase the nucleation density of the isolated graphene domains for the analysis. Only two grain boundaries from $\mathrm{Cu}$ are visible near the corners of the view field. The center region (region 1) between two $\mathrm{Cu}$ grain boundaries clearly demonstrates the high efficiency of $\mathrm{Cu}$ surface monocrystallization up to centimeter scale. The information about the size and orientation of more than 200 aHGDs grown on this $\mathrm{Cu}$ surface within these three regions is obtained and shown in Fig. $3 \mathrm{~b}$ and c, respectively. The diagonal sizes of these domains range from 0.1 to $1.1 \mathrm{~mm}$ and are fitted well by Gaussian distribution with a center value of $\sim 0.57 \mathrm{~mm}$. Considering that all the aHGDs have a hexagonal shape, it is characterized by the orientation angle $\theta$ relative to the horizontal line in the OM images. For all the aHGDs shown in Fig. $2 \mathrm{~b}$, their dominant orientation angles are $10^{\circ}, 1^{\circ}$, and $40^{\circ}$ and solely observed in the region 1 , region 2 , and region 3 (Fig. S4, ESI $\dagger$ ), respectively. Especially, among all the 177 graphene domains measured in the region 1 , only 3 of them exhibit different orientation angles other than $10^{\circ}$, corresponding to a unidirectional domain alignment of $>98 \%$. The abovementioned result also confirms a strict alignment of the aHGDs over a distance exceeding $1 \mathrm{~cm}$ scale, and similar results can also be obtained from the graphene domains in the region 2 and 3.

The unidirectional alignment of these aHGDs suggests a possibility of seamless stitching when they coalesce, i.e., to form a scalable single-crystalline graphene film. Therefore, it is of great significance to characterize the coalescence interfaces for the aHGDs. For this purpose, atomic force microscopy (AFM) analysis on the aligned and misaligned graphene domains and their coalescence interfaces was conducted. As shown in Fig. 4, a coalescence corner (region A in Fig. 4b) by two aHGDs is chosen for the lateral force (friction), current, and deflection (error signal) measurements, and the results are presented in Fig. 4c. Except for the regular terraces of the underlying $\mathrm{Cu}$ substrate, all signals are rather homogeneous within the domains as well as at the coalescence interface where a domain boundary is supposed to appear if any. By contrast, for a coalescence interface formed by two misaligned graphene domains, the clearly enhanced signals of lateral force, current, and deflection are detected, as shown in Fig. S5 in ESI. $\dagger$ Apparently, the contrasts of lateral force, current, and deflection signals at the coalescence interface of two graphene domains are associated with the presence of domain boundaries or overlapped step edges, resulted from the differences in the structural and physical properties at these domain coalescence interfaces. The AFM measurement results for the coalesced domains with seamless stitching can be routinely reproduced
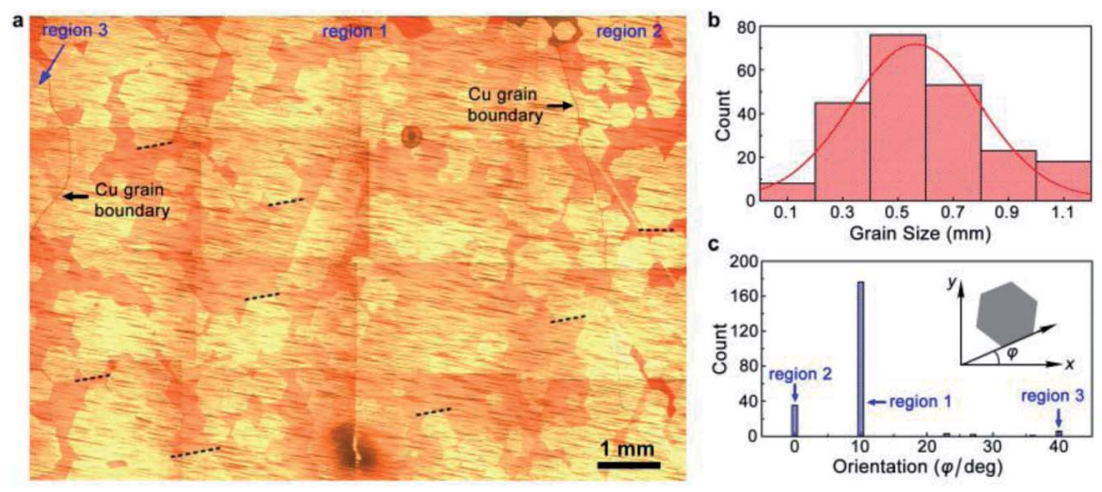

Fig. 3 (a) The obtained OM image of aHGDs on the Cu surface over $\sim 1 \mathrm{~cm}^{2}$ scanned area (growth time: 30 min). Only two Cu grain boundaries are visible in the image, demonstrating the high efficiency of oxide-assisted surface monocrystallization. (b) Histogram of the size distribution for aHGDs in (a), which follows Gaussian distribution. (c) Histogram of the orientation distribution for aHGDs in (a). Three different domain orientations are found in the three $\mathrm{Cu}$ grains, and only less than $1 \%$ graphene domains are misoriented in the same Cu grain. 
for the aligned interfaces (Fig. S6, ESI $\dagger$ ). We have also performed the atomic-resolved AFM measurements to reveal the crystalline orientation of two aHGDs. As seen in Fig. $4 d$, a hexagonal lattice with a lattice constant of $\sim 0.25 \mathrm{~nm}$ can be extracted from the data, showing a perfect agreement with the known lattice constant of monolayer graphene $(0.246 \mathrm{~nm}){ }^{23}$ and it is easy to derive that two aHGDs have the same lattice orientation. Moreover, as shown in Fig. 4e, we have indicated the zigzag directions by arrows, and apparently, the edges of both aHGDs are along this direction; this is believed to result from the metal-assisted $\mathrm{H}_{2}$ anisotropic etching process. This zigzag edge structure is also consistent with the low D-band intensities measured from the aHGD edges shown in Fig. 2.

Finally, we have further discussed the role of the $\mathrm{Cu}$ surface oxide layer in the growth of millimeter-sized aHGDs. We have conducted several control experiments using the conditions of (a) with and without the surface oxide and (b) heating and annealing with and without $\mathrm{H}_{2}$. As shown in Table $\mathrm{S} 1$ and Fig. S7 in ESI, $\dagger$ with surface oxide, the graphene domains with significantly increased sizes can be obtained after growth. This can be attributed to the fact that the $O$ residues can be embedded into the $\mathrm{Cu}$ surface to make it featureless after the reduction. ${ }^{22}$ However, the domain alignment is only possible when $\mathrm{H}_{2}$ is introduced during the annealing process at high temperatures. This suggests that if it is not removed during the heating process, the pre-treated surface oxide layer can help to reconstruct $^{23}$ and monocrystallize the $\mathrm{Cu}$ surface under the reduction gas atmosphere at high temperatures; this is probably due to the enhanced pre-melting effect ${ }^{24,25}$ near the $\mathrm{Cu}$ surface resulting from the adsorbed oxygen species to reconstruct the $\mathrm{Cu}$ surface for the more stable (111) textures. Moreover, the CVD growth of aHGDs with different periods, as shown in Fig. S8 in ESI, $\uparrow$ shows that during the first several minutes of growth, only few domains nucleate on the $\mathrm{Cu}$ surface. This may also suggest a period for the $\mathrm{Cu}$ surface reconstruction by the

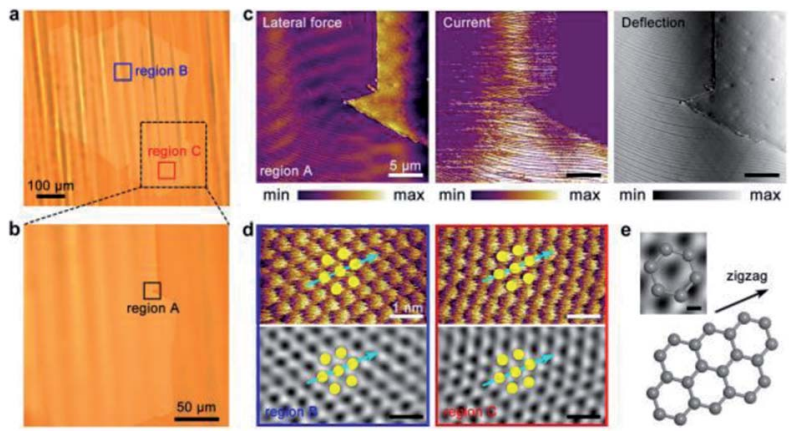

Fig. 4 ( $a$ and b) OM images of the two coalesced aHGDs and their interface, respectively. (c) AFM measurements of lateral force (friction), current, and deflection (error signal) scanned from the region A of the aHGD interface in (b). No apparent signal differences are detected at the interface in all these images. (d) Atomic-resolution AFM images and their corresponding Fourier transform images from the region $B$ and $C$ in (a). The lattices in both regions exhibit the same orientation, demonstrating the unidirectional alignment of the aHGDs. (e) Lattice orientation detected from atomic-resolution AFM confirms the zigzag structure of the aHGD edges. Scale bar in (e): $0.1 \mathrm{~nm}$. sudden introduction of $\mathrm{H}_{2}$, which is not suitable for graphene nucleation and growth. The domains later exhibit a fast rate of $\sim 30 \mu \mathrm{m} \mathrm{min}{ }^{-1}$, much faster than that without the surface oxide layer; this demonstrates that the $\mathrm{Cu}$ surface oxide layer can provide surface oxygen to accelerate the growth of graphene domains. ${ }^{11}$ It needs to be mentioned that although previous literature has shown that large-scale single-crystal surfaces and graphene domains can be obtained by annealing under an $\mathrm{Ar}$ atmosphere, ${ }^{18,22,26}$ the results in Fig. S7† clearly demonstrate the importance of both pre-treated $\mathrm{Cu}$ surface oxide layer and annealing under a non-reduction gas atmosphere in the enlargement and alignment of the graphene domains through the $\mathrm{Cu}$ surface monocrystallization.

\section{Conclusion}

In summary, we have demonstrated the CVD growth of scalable single-crystalline graphene by seamlessly stitching millimetersized unidirectional aHGDs on different types of commercially available $\mathrm{Cu}$ foils without any repeated substrate polishing and/ or hydrogen annealing processes. The key factor for the growth is the surface reconstruction and monocrystallization of the $\mathrm{Cu}$ foils achieved by pre-introducing an oxide layer and the sequential Ar annealing. The aHGD growth shows an average growth rate of $>20 \mu \mathrm{m} \mathrm{min}^{-1}$ and a misorientation possibility of $<2 \%$. The AFM lateral force, current, and deflection measurements present no differences at the domain coalescence interfaces; this clearly demonstrates the elimination of domain boundaries and seamless stitching of the domains. Compared with that reported in previous studies, the average size for our hexagonal graphene domains is enlarged by 1-2 orders of magnitude (from tens of micrometers to millimeter); this further eliminates the formation of domain boundaries. We believe that this cost-effective and time-saving synthetic method for large-scale single-crystalline graphene will be of significant attraction presenting valuable guidance for future industrial devices and applications of high-quality graphene.

\section{Experimental}

\section{Graphene synthesis}

A piece of commercially available $\mathrm{Cu}$ foils (\#46365, \#13382, and \#42972 from Alfa Aesar China Chemical Co., Ltd., \#113243 from Nilaco Co., Ltd., etc.) was heated at $200-300^{\circ} \mathrm{C}$ in the air by a hot plate for 1-30 min to create a layer of metal oxide before it was loaded into a CVD quartz chamber. For the growth of millimeter-sized aligned hexagonal graphene domains (aHGDs), an atmospheric pressure CVD process was used. The chamber temperature was increased to $1060^{\circ} \mathrm{C}$ and maintained (total time: $70 \mathrm{~min})$ under an atmosphere of $\operatorname{Ar}(1000 \mathrm{sccm})$ to anneal and monocrystallize the $\mathrm{Cu}$ surface, and the pressure inside the chamber was kept at $1 \mathrm{~atm}$. For graphene growth, 20 sccm diluted $\mathrm{CH}_{4}(0.05 \%$ in $\mathrm{Ar}), 10 \mathrm{sccm} \mathrm{H}$, and $300 \mathrm{sccm} \mathrm{Ar}$ were employed. After being cooled down to room temperature, the as-grown graphene on the $\mathrm{Cu}$ foil was heated in the air at $160{ }^{\circ} \mathrm{C}$ for $10 \mathrm{~min}$ for direct visualization. ${ }^{20}$ 


\section{Characterizations}

The characterizations of graphene were carried out by SEM (3 kV, S-3400, Hitachi Co., Ltd.), XRD (X'Pert PRO, PANalytical Co., Ltd.), micro-Raman spectroscopy (532 nm wavelength excitation laser, LabRAM HR Evolution, Horiba Co., Ltd.), OM (yellow light by Shanghai 8XB-PC from Shanghai Optical Instrument Factory and blue light by Olympus BXFM-ILHS from Olympus Co., Ltd), EBSD using scanning auger electron spectroscopy (AES, PHI710, ULVAC-PHI Inc.), AFM (Multimode 8 system equipped with a silicon probe SNL-10 from Bruker Co., Ltd.), and highresolution AFM/STM (NT-MDT spectrum Instruments).

\section{Conflicts of interest}

There are no conflicts to declare.

\section{Acknowledgements}

A part of this work was financially supported by the National Key Scientific Instruments and Equipment Development Project of China (61427901), the National Science Foundation of China (11502231, 11621062, 61471317), and the Fundamental Research Funds for the Central Universities (2017QNA4032).

\section{References}

1 X. S. Li, W. W. Cai, J. H. An, S. Kim, J. Nah, D. X. Yang, R. Piner, A. Velamakanni, I. Jung, E. Tutuc, S. K. Banerjee, L. Colombo and R. S. Ruoff, Science, 2009, 324, 1312-1314.

2 S. Bae, H. Kim, Y. Lee, X. Xu, J.-S. Park, Y. Zheng, J. Balakrishnan, T. Lei, H. Ri Kim, Y. I. Song, Y.-J. Kim, K. S. Kim, B. Ozyilmaz, J.-H. Ahn, B. H. Hong and S. Iijima, Nat. Nanotechnol., 2010, 5, 574-578.

3 X. Li, L. Colombo and R. S. Ruoff, Adv. Mater., 2016, 28, 62476252.

4 P. Y. Huang, C. S. Ruiz-Vargas, A. M. van der Zande, W. S. Whitney, M. P. Levendorf, J. W. Kevek, S. Garg, J. S. Alden, C. J. Hustedt, Y. Zhu, J. Park, P. L. McEuen and D. A. Muller, Nature, 2011, 469, 389-392.

5 L. Tapasztó, P. Nemes-Incze, G. Dobrik, K. Jae Yoo, C. Hwang and L. P. Biró, Appl. Phys. Lett., 2012, 100, 053114.

6 Z. Song, V. I. Artyukhov, B. I. Yakobson and Z. Xu, Nano Lett., 2013, 13, 1829-1833.

7 Z. Fei, A. S. Rodin, W. Gannett, S. Dai, W. Regan, M. Wagner, M. K. Liu, A. S. McLeod, G. Dominguez, M. Thiemens, H. Castro Neto Antonio, F. Keilmann, A. Zettl, R. Hillenbrand, M. M. Fogler and D. N. Basov, Nat. Nanotechnol., 2013, 8, 821-825.

8 X. Li, C. W. Magnuson, A. Venugopal, R. M. Tromp, J. B. Hannon, E. M. Vogel, L. Colombo and R. S. Ruoff, J. Am. Chem. Soc., 2011, 133, 2816-2819.
9 Z. Yan, J. Lin, Z. Peng, Z. Sun, Y. Zhu, L. Li, C. Xiang, E. L. Samuel, C. Kittrell and J. M. Tour, ACS Nano, 2012, 6, 9110-9117.

10 H. Zhou, W. J. Yu, L. Liu, R. Cheng, Y. Chen, X. Huang, Y. Liu, Y. Wang, Y. Huang and X. Duan, Nat. Commun., 2013, 4, 2096.

11 Y. Hao, M. S. Bharathi, L. Wang, Y. Liu, H. Chen, S. Nie, X. Wang, H. Chou, C. Tan, B. Fallahazad, H. Ramanarayan, C. W. Magnuson, E. Tutuc, B. I. Yakobson, K. F. McCarty, Y.-W. Zhang, P. Kim, J. Hone, L. Colombo and R. S. Ruoff, Science, 2013, 342, 720-723.

12 X. Chen, P. Zhao, R. Xiang, S. Kim, J. Cha, S. Chiashi and S. Maruyama, Carbon, 2015, 94, 810-815.

13 V. L. Nguyen, B. G. Shin, D. L. Duong, S. T. Kim, D. Perello, Y. J. Lim, Q. H. Yuan, F. Ding, H. Y. Jeong, H. S. Shin, S. M. Lee, S. H. Chae, Q. A. Vu, S. H. Lee and Y. H. Lee, Adv. Mater., 2015, 27, 1376-1382.

14 V. L. Nguyen and Y. H. Lee, Small, 2015, 11, 3512-3528.

15 H. Wang, X. Xu, J. Li, L. Lin, L. Sun, X. Sun, S. Zhao, C. Tan, C. Chen, W. Dang, H. Ren, J. Zhang, B. Deng, A. L. Koh, L. Liao, N. Kang, Y. Chen, H. Xu, F. Ding, K. Liu, H. Peng and Z. Liu, Adv. Mater., 2016, 28, 8968-8974.

16 L. Sun, L. Lin, J. Zhang, H. Wang, H. Peng and Z. Liu, Nano Res., 2017, 10, 355-363.

17 M. Zeng, L. Tan, L. Wang, R. G. Mendes, Z. Qin, Y. Huang, T. Zhang, L. Fang, Y. Zhang, S. Yue, M. H. Rümmeli, L. Peng, Z. Liu, S. Chen and L. Fu, ACS Nano, 2016, 10, 7189-7196.

18 J. Hu, J. Xu, Y. Zhao, L. Shi, Q. Li, F. Liu, Z. Ullah, W. Li, Y. Guo and L. Liu, Sci. Rep., 2017, 7, 45358.

19 X. Xu, Z. Zhang, J. Dong, D. Yi, J. Niu, M. Wu, L. Lin, R. Yin, M. Li, J. Zhou, S. Wang, J. Sun, X. Duan, P. Gao, Y. Jiang, X. Wu, H. Peng, R. S. Ruoff, Z. Liu, D. Yu, E. Wang, F. Ding and K. Liu, Sci. Bull., 2017, 62, 1074-1080.

20 C. Jia, J. Jiang, L. Gan and X. Guo, Sci. Rep., 2012, 2, 707.

21 A. C. Ferrari, J. C. Meyer, V. Scardaci, C. Casiraghi, M. Lazzeri, F. Mauri, S. Piscanec, D. Jiang, K. S. Novoselov, S. Roth and A. K. Geim, Phys. Rev. Lett., 2006, 97, 187401.

22 J. Li, X. Wang, X. Liu, Z. Jin, D. Wang and L. Wan, J. Mater. Chem. C, 2015, 3, 3530.

23 A. Y. Lu, S. Y. Wei, C. Y. Wu, Y. Hernandez, T. Y. Chen, T. H. Liu and Z. Y. Juang, RSC Adv., 2012, 2, 3008.

24 J. W. M. Frenken and J. F. v. d. Veen, Phys. Rev. Lett., 1985, 54, 134-137.

25 Z.-J. Wang, G. Weinberg, Q. Zhang, T. Lunkenbein, A. KleinHoffmann, M. Kurnatowska, M. Plodinec, Q. Li, L. Chi, R. Schloegl and M.-G. Willinger, ACS Nano, 2015, 9, 15061519.

26 L. Gan and Z. Luo, ACS Nano, 2013, 7, 9480-9488. 